\title{
Excessive Early Pregnancy Weight Gain and Risk of Gestational Diabetes Mellitus
}

\author{
${ }^{1}$ Maya Menon, ${ }^{2} \mathrm{~K}$ Vasantha
}

\section{ABSTRACT}

Objectives: To study the association between excessive early pregnancy weight gain and risk of gestational diabetes mellitus (GDM).

Materials and methods: Study design-prospective observational study done at ESIC Medical College \& PGIMSR, Chennai during the period November 2013 to May 2015. A total of 250 women who attended antenatal outpatient department at ESI Hospital Chennai were recruited for the study. Height and weight of the pregnant women was recorded during their first visit (up to 6 weeks) and at 14 weeks of gestation. Body mass index (BMI) was calculated according to Quetelet index. Waist hip ratio was measured at 6 weeks. The early gestation weight gain more than $2 \mathrm{~kg}$ was considered as excessive weight gain and was associated to the risk of developing GDM. Blood sugar was estimated using Diabetes in Pregnancy Study Group Index (DIPSI) standards.

Results: Of the 250 women studied, 104 women developed GDM. Excessive weight gain in the 1st trimester of pregnancy was significantly associated with the development of GDM with a $p$-value of 0.000 . The other risk factors studied were BMI $(p=0.0064)$, waist/hip ratio $(p=0.0015)$, family history of diabetes mellitus $(D M)(p=0.00)$, which also had a significant association.

Clinical significance: This study shows that excessive weight gain in 1st trimester had an increased risk of GDM. The benefit of early identification of excess gestational weight gain would be the opportunity for intervention early in pregnancy to ideally decrease GDM.

Conclusion: Excessive early pregnancy weight gain, primarily in the 1st trimester may increase the risk of developing GDM. Thus, preventing excessive early pregnancy weight gain in the 1st trimester can be modified by lifestyle changes and simple exercise, which is a costless intervention, and can also decrease maternal and neonatal morbidity effectively.

Keywords: Body mass index, Excessive weight gain, First trimester, Gestational diabetes mellitus, Waist/hip ratio.

How to cite this article: Menon M, Vasantha K. Excessive Early Pregnancy Weight Gain and Risk of Gestational Diabetes Mellitus. J South Asian Feder Obst Gynae 2016;8(3):189-192.

Source of support: Nil

Conflict of interest: None

\footnotetext{
${ }^{1}$ Associate Professor, ${ }^{2}$ Postgraduate

1,2Department of Obstetrics and Gynecology, ESIC Medical College \& PGIMSR, Chennai, Tamil Nadu, India

Corresponding Author: Maya Menon, Associate Professor Department of Obstetrics and Gynecology, ESIC Medical College \& PGIMSR, Chennai, Tamil Nadu, India, Phone: +919444477791, e-mail: mayasree@gmail.com
}

Date of received: 12 May 2016

Date of acceptance: 10 June 2016

Date of publication: July 2016

\section{INTRODUCTION}

The amount of weight gain during pregnancy can affect the immediate and future health of a woman and her infant. It is known that there is an association between excessive gestational weight gain and increased birth weight and postpartum weight retention. In 2009, US Institute of Medicine (IOM) ${ }^{1}$ published revised gestational weight gain guidelines that are based on prepregnancy body mass index (BMI) ranges for underweight, normal weight, overweight, and obese women, which is also recommended by World Health Organization (WHO). This is independent of age, parity, smoking, history, race, and ethnic background. As per the guidelines in 1st trimester, $>2 \mathrm{~kg}$ is considered as excessive early gestational weight gain.

The term gestational diabetes mellitus (GDM) was first coined by O Sullivan in 1961. Seshiah et $\mathrm{al}^{2}$ in a prospective study screened and diagnosed GDM in $17.8 \%$ women of urban population, $13.8 \%$ of semi-urban population, and $9.9 \%$ of rural. Indian women reported highest frequency of GDM, compared to other ethnic groups in South Asian countries. The cause for raising prevalence rate in developing countries was related to urbanization, sedentary lifestyle, dietary pattern change, and raising prevalence of obesity. Recent multicentric study $^{3}$ in India showed highest prevalence of GDM in Tamil Nadu and lowest in Kashmir.

Researchers have stated that "Avoidance of excessive weight gain in early pregnancy" is an efficient method to prevent GDM and its complications. ${ }^{4-7}$ It is observed that "Indian bodies and genetic composition differ from their western counterparts as Indians have abdominal obesity compared to the western people whose body is uniformly obese and fix the Indians as high risk for hypertension and diabetes mellitus (DM)." 8

Excessive pregnancy weight gain is an important risk factor for developing GDM. Not many studies exist that observe an increase in weight gain in early pregnancy and occurrence of GDM in Indian population. Hence 
we aimed at finding an association between excessive early pregnancy weight gain in the 1st trimester and development of GDM.

In our study the aim was to find out the correlation between excessive early gestational weight gain (GWG) and risk of GDM and its related maternal and fetal complication. According to IOM (2009) guidelines, calculations assume a 1.1 to $4.4 \mathrm{lb}(2 \mathrm{~kg})$ recommended weight gain in 1st trimester. According to Asian Indian guidelines, waist circumference cutoff of Indian men is $90 \mathrm{~cm}$ (102 globally) and for Indian women is $80 \mathrm{~cm}$ (88 cm globally).

\section{MATERIALS AND METHODS}

This is a prospective observational study done in pregnant women attending antenatal outpatient (OP) department at ESIC Medical College between November 2013 and May 2015. Permission was obtained from the Institute Ethical Committee. Informed written consent was taken from the patients. Sample size was calculated as 250 using qualitative analysis. Anticipating dropouts, 285 women who attended antenatal OP department at ESI hospital were recruited for the study. Out of 285 women, 12 antenatal women lost follow-up, 8 women prepregnant weight report were not available, and 15 women developed obstetrics complications like anemia, hypothyroidism, and molar pregnancy. Women with singleton pregnancy, on regular antenatal checkup, and no associated comorbidity were included in the study. Women with multiple pregnancy, anemia, chronic hypertension, pregestational DM, thyroid disorders, and molar pregnancy were excluded from the study. Maternal weight gain from prepregnancy weight (6 weeks) to 14 weeks gestation was measured on a weighing scale that could measure nearest to $0.1 \mathrm{~kg}$.

During 1st antenatal visit height, weight, and waist/ hip ratio were measured. Body mass index was calculated using Quetelet index. Categorization was done according to Asian Indian BMI guidelines. Detailed history, general examination, and obstetric examination were done. Under sterile aseptic precautions, blood samples $(6-8 \mathrm{~mL})$ were drawn for hemoglobin, glycosylated hemoglobin, oral glucose challenge test (OGCT). Since fetal beta cells starts secreting insulin by 9 to 11 weeks, OGCT was done at 14 weeks. Follow-up was done monthly till 28 weeks, fortnightly till 36 weeks, weekly till term, and each time weight was measured. If patient was not diagnosed as GDM in 1st trimester, OGCT was repeated at 24 to 28 and 32 to 34 weeks gestation. If diagnosed as GDM, the patient was treated as per protocol ${ }^{9}$ and followed up till delivery.

Standardized Diabetes in Pregnancy Study Group Index (DIPSI) protocol was used to estimate the blood sugar. It is a single-step procedure, 75 gm of glucose was given orally, and blood collected after 2 hours. If blood sugar was more than $140 \mathrm{gm} / \mathrm{dL}, \mathrm{GDM}$ was diagnosed. This procedure is approved by Ministry of Health, Government of India and recommended by WHO.

According to Cunningham et $\mathrm{al}^{9}$ weight gain recommendations and IOM (2009) guidelines, which was endorsed by ACOG (2012), GWG $>2 \mathrm{~kg}$ (upper limit of IOM (2009) for 1st trimester) till 14 weeks was taken as excessive early GWG in our study.

Data analysis was done using Statistical Package for the Social Sciences (SPSS) version 17. Chi-square test was used to test the significance of association between family history of DM and GDM. Early pregnancy weight gain, $\mathrm{BMI}$, waist/hip ratio, and development of GDM were analyzed using Student's t-test. p-value $<0.05$ denoted significant relationship.

\section{RESULTS}

A total of 285 women who attended antenatal OP department in ESIC were recruited for the study. Out of 285 women, 12 antenatal women lost follow-up, 8 prepregnancy weight report of women were not available, and 15 developed obstetrics complication like anemia, hypothyroidism, and molar pregnancy. The mean age of women who developed GDM was 27.5 \pm 4 years and without GDM were $26.2 \pm 3.9$ years in the study. Of the 250 women studied, 104 women developed GDM who were compared with control of 146 women belonging to non-GDM group. There was no significant association demonstrated between parity and GDM. Table 1 shows that BMI and GDM had a significant association with $80 \%$ obese women and $60 \%$ overweight women developing GDM. Table 2 denotes that women with abnormal waist/ hip ratio developed GDM: $73 \%$ of women with abnormal

Table 1: Body mass index and GDM

\begin{tabular}{llllll}
\hline & \multicolumn{3}{c}{ Gestational diabetes mellitus } \\
\cline { 2 - 4 } \cline { 2 - 3 } \cline { 6 - 7 } Body mass index $(n)$ & $n$ & $\%$ & & & \multicolumn{2}{c}{ No } \\
\cline { 2 - 3 } Underweight $(\leq 18.4)(4)$ & 0 & 0 & & 4 & 100.0 \\
Normal $(18.5-22.9)(185)$ & 67 & 36.2 & & 118 & 63.8 \\
Overweight $(23-24.9)(35)$ & 21 & 60.0 & & 14 & 40.0 \\
Obese $(\geq 25)(26)^{*}$ & 21 & 80.8 & & 5 & 19.2 \\
\hline
\end{tabular}

${ }^{*} p=0.006$ significance

Table 2: Waist/hip ratio \% (at 6 weeks) and GDM

\begin{tabular}{llllll}
\hline & \multicolumn{3}{c}{ Gestational diabetes mellitus } \\
\cline { 2 - 3 } \cline { 6 - 6 } \cline { 5 - 6 } Waist/hip ratio \% $(n)$ & $n$ & & & \multicolumn{2}{c}{ No } \\
\cline { 2 - 3 } \cline { 5 - 6 } Normal (220) & 83 & 37.7 & & 137 & 62.3 \\
Abnormal (30) & $22^{*}$ & 73.0 & & 8 & 27.0 \\
\hline
\end{tabular}

${ }^{*} p=0.001$ significance 
Excessive Early Pregnancy Weight Gain and Risk of Gestational Diabetes Mellitus

\begin{tabular}{|c|c|c|c|c|}
\hline \multirow[b]{3}{*}{ Family history of DM (n) } & \multicolumn{4}{|c|}{ Gestational diabetes mellitus } \\
\hline & \multicolumn{2}{|c|}{ Yes } & \multicolumn{2}{|c|}{ No } \\
\hline & $n$ & $\%$ & $n$ & $\%$ \\
\hline Yes (83) & $68^{*}$ & 56.6 & 15 & 43.4 \\
\hline No (167) & 36 & 40.7 & 131 & 59.3 \\
\hline
\end{tabular}

waist/hip ratio developed GDM in our study. Table 3 shows the relationship between family history of DM and development of GDM in which $56.6 \%$ of women developed GDM who had a positive family history for DM. Table 4 shows the relation between early pregnancy weight gain and GDM, according to which $59.6 \%$ women who had an increase in weight in 1st trimester developed GDM which was statistically significant.

\section{DISCUSSION}

In 2009, the US IOM published revised guidelines for GWG. The goal of these guidelines is to optimize both maternal and neonatal outcomes. Hedderson et $\mathrm{al}^{10}$ in a multiethnic cohort of 345 women with GDM found an $82 \%$ increase in women who developed GDM if the rate of weight gain in 1st trimester was between 0.27 and 1.9 $\mathrm{kg} /$ week. Zilko et al ${ }^{11}$ collected data from 4,496 births during 1979 in the National Long Survey of Youth and described the correlation between GWG and increased rates of GDM, lower segment cesarean section (LSCS), postpartum weight retention, large for gestational age as well as childhood overweight. They concluded that $40 \%$ of women with excessive GWG retained greater than $2.5 \mathrm{~kg}$ from 12 to 24 months after delivery and $29 \%$ of children had a BMI more than 85th percentile. They investigated the association between excessive early GWG and the risk of GDM and they demonstrated the stronger association between weight gain during the 1st trimester and GDM. We have observed that early pregnancy weight gain was significantly associated with the risk of developing GDM which is obvious from our results in which 59.6\% women developed GDM.

Our findings were similar to other studies ${ }^{12,13}$ who have found a significant association between overweight, obese women, and GDM. Women with normal BMI, overweight, and obese who developed GDM were 36.2, 60 , and $80.8 \%$ respectively in our study.

Hedderson et $\mathrm{al}^{10}$ stated "As all pregnancy progress, an increase in insulin resistance occurs" due to decreased beta-cell capacity caused by excessive weight gain and an increased weight gain during 1st trimester will thrust them into GDM. They observed that the waist circumference of $85.5 \mathrm{~cm}$ (sensitivity $75 \%$, specificity
Table 4: Gestational diabetes mellitus and early pregnancy weight gain

\begin{tabular}{llllll}
\hline \multirow{2}{*}{$\begin{array}{l}\text { Gestational diabetes } \\
\text { mellitus }\end{array}$} & \multicolumn{3}{c}{ Normal } & & \multicolumn{2}{c}{ Abnormal } \\
\cline { 2 - 3 } & $n$ & & & $n$ & $\%$ \\
\hline Yes $(104)^{*}$ & 42 & 40.4 & & $62^{*}$ & 59.6 \\
No $(146)$ & 120 & 82.2 & & 26 & 17.8 \\
\hline${ }^{*} \mathrm{p}<0.000$ significance & & & &
\end{tabular}

$81.4 \%$ ) and BMI of $24.3 \mathrm{~kg} / \mathrm{m}^{2}$ (sensitivity $75 \%$, specificity $86.5 \%$ ) had the best predictive value of developing GDM.

Basraon et $\mathrm{al}^{14}$ and Madhavan et $\mathrm{al}^{15}$ showed that the prevalence of GDM was higher in pregnant women with abnormal waist/hip ratio. Similarly we have observed that $73 \%$ women with abnormal waist/hip ratio developed GDM.

Retnakaran et $\mathrm{al}^{16}$ in a study concluded that there is a relationship between family history of diabetes and GDM. In our study, $56.6 \%$ of the women with family history of DM developed GDM which was statistically significant.

Excessive early pregnancy weight gain, primarily in the 1st trimester may increase a women's risk of GDM and its related maternal and fetal complications. It can be represented as a modifiable risk factor occurring during early pregnancy. Lifestyle modifications like simple exercises (walking) and dietary modifications can prevent GDM and its related complications. This costless early intervention can make future generations healthy.

\section{CLINICAL SIGNIFICANCE}

This study shows that excessive weight gain in 1st trimester had an increased risk of GDM. A benefit of early identification of excess GWG would be the opportunity for intervention early in pregnancy to ideally decrease GDM.

\section{CONCLUSION}

Identifying excessive GWG, early in gestation, could allow the opportunity to intervene at an earlier gestational age with behavioral modification, such as nutrition and exercise counseling and potentially decrease the rate of adverse maternal and neonatal outcome.

\section{REFERENCES}

1. Institute of Medicine, National Academy of Sciences. Nutrition during pregnancy. Washington, DC: National Academy Press; 2009.

2. Seshiah V, Balaji V, Balaji MS, Paneerselvam A, Arthi T, Thamizharasi M, Datta M. Prevalence of gestational diabetes mellitus in South India (Tamil Nadu): a community based study. J Assoc Physicians India 2008 May;56:329-333. 
3. Seshiah V, Das AK, Balaji V, Joshi SR, Parikh MN, Gupta S. Diabetes in Pregnancy Study Group. Gestational diabetes mellitus guidelines. J Assoc Physicians India 2006 Aug;54: 622-628.

4. Metzger JJ. Excessive gestational weight gain in women without gestational diabetes mellitus is associated neonatal fat mass and hyperinsulinemia. Endocr Rev 2011;32: 234-238.

5. Stein J, et al. Early pregnancy weight gain linked to gestational diabetes. Los Angeles Times Medicine, February 23, 2010.

6. Herring SJ, Oken E, Rifas Shiman SL, Rich Edwards JW, Stuebe AM, Kleinman KP, Gillman MW. Weight gain in pregnancy and risk of maternal hyperglycemia. Am J Obstet Gynecol 2009 Jul;201(1):61.e61-61.e67.

7. Aziz N, Kallur SD, Nirmalan PK. Implications of the revised consensus body mass indices for Asian Indians on clinical obstetric practice. J Clin Diagn Res. 2014 May;8(5):OC01-OC03.

8. Carreno CA, Clifton RG, Hauth JC, Myatt L, Roberts JM, Spong CY, Varner MW, Thorp JM Jr, Mercer BM Peaceman AM, et al. Excessive early gestational weight gain and risk of gestational diabetes mellitus in nulliparous women. Obstet Gynecol 2012 Jun;119(6):1127-1233.

9. Cunningham FG, Leveno KJ, Steven L, Bloom SL. Williams text book of obstetrics. 24th ed. New York: Mc-Graw Hill Education; Vol. 9; 2014. p. 172-177.

10. Hedderson MM, Gunderson EP, Ferrara A. Gestational weight gain and risk of gestational diabetes mellitus. Obstet Gynecol 2010 Mar;115(3):597-604.
11. Margerison Zilko CE, Rehkopf D, Abrams B. Association of maternal gestational weight gain with short and long term maternal and child health outcomes. Am J Obstet Gynecol 2010 Jun;202(6):574.e1-574.e8.

12. Whiteman VE, Aliyu MH, August EM, McIntosh C, Duan J, Alio AP, Salihu HM. Changes in prepregnancy body mass index between pregnancies and risk of gestational and type 2 diabetes. Arch Gynecol Obster 2011 Jul;284(1):235-240.

13. Alberico S, Montico M, Barresi V, Monasta L, Businelli C, Soini V, Erenbourg A, Ronfani L, Maso G; Multicentre Study Group on Mode of Delivery in Friuli Venezia Giulia. The role of gestational diabetes, pre pregnancy body mass index and gestational weight gain on the risk of newborn macrosomia: Results from a prospective multicentre study. BMC Pregnancy Childbirth 2014 Jan;14:23.

14. Basraon SK, Mele L, Myatt L, Roberts JM, Hauth JC, Leveno KJ, Varner MW, Wapner RJ, Thorp JM Jr, Peaceman AM et al. Relationship of early pregnancy waist hip ratio versus body mass index with gestational diabetes mellitus and insulin resistance. Am J Perinatol 2016 Jan;33(1):114-121.

15. Madhavan A, Beena Kumari R, Sanal MG. A Pilot study on the usefulness of body mass index and waist hip ratio as a predictive tool for gestational diabetes in Asian Indians. Gynecol Endocrinol 2008 Dec;24(12):701-707.

16. Retnakaran R, Qi Y, Sermer M, Connelly PW, Zinman B, Hanely AJ. Isolated hyperglycemia at 1 hour on oral glucose tolerance test in pregnancy resembles gestational diabetes mellitus in predicting postpartum metabolic dysfunction. Diabetes Care 2008 Jul;31(7):1275-1281. 